\title{
THE TRANSIENT AUDITORY EVOKED GAMMA-BAND FIELD
}

\author{
Christo Pantev and Thomas Elbert
}

Institute of Experimental Audiology, University of Münster, Germany

\section{INTRODUCTION}

A stimulus elicits an afferent volley of action potentials, which is transmitted to the brain where it generally evokes cooperative neural mass activity. This means that transient sensory stimulation will, locally in time and space, enhance average neural depolarization which results in the slow event-related potentials (Elbert, 1993). Furthermore, coordinated patterns of neural mass activity will result in fluctuations in the gamma band, i.e. in the frequency range above $20-30 \mathrm{~Hz}$. The overall sum of depolarizations manifests itself in EEG and MEG, and the corresponding fluctuations are referred to as induced rhythms, a term which was introduced to call attention to a large variety of oscillatory responses that follow either clearly timed stimuli or less sharply timed state changes such as attention, sleep, expectations and seizures. Induced rhythms may be tightly or loosely time-locked to the triggering event and, hence, may or may not appear in averaged responses or only in single trials. Bullock, in his chapter of this book, suggested considering evoked rhythms as a subset of induced activity. Evoked rhythms follow clearly timed stimuli or events. They are more or less tightly time-locked and thus may be extracted by conventional averaging across many responses, i.e. by averaging in the time domain. The term evoked is generally not used for responses to less sharply timed state changes or events such as those just named. There is, however, no sharp border between the two terms, and therefore it may be useful to add descriptors, such as "phase locked" or "nonphase locked" etc.

The evoked gamma-band activity is stimulus- and phase-locked and thus it can be extracted from the noise in the time-domain using the averaging procedure. The present chapter reports results from studying the evoked, i.e. the time-locked transient oscillatory gamma-band activity, arising from the auditory cortex in humans.

The first oscillatory activity in the $40-\mathrm{Hz}$ range arising from the auditory cortex and the hippocampus of the cat as response to auditory stimulation was recorded by Basar (1972; Basar and Özesmi (1972); Basar and Ungan, (1973) and investigated in the following years by Basar and his group (Basar et al., 1987; Basar, 1988; Basar- 
Eroglu and Basar, 1991). Human auditory evoked steady state responses in the gamma-band were intensively studied by Galambos et al. (1981); Mäkelă and Hari (1987); Galambos and Makeig (1988); Hari et al. (1989); Ribari et al. (1989) and Romani et al. (1982). Both cortical and subcortical sources for the steady-state responses have been proposed (Mäkelä and Hari, 1987). Recently transient electric and magnetic gamma-band responses time locked to the onset of auditory stimuli that increases in amplitude as stimulus rate is decreased below one per second and their sources located in the auditory cortex have been shown (Makeig and Galambos, 1989; Pantev et al., 1991; Pantev et al., 1993).

The goal of the present study was to increase our understanding of the transient auditory gamma-band responses (GBR) as well as the function that GBR may serve for sensory processing, particularly in the human auditory cortical areas. Therefore, we carried out several parametric and attentional studies. The functional behavior of the GBR in the time domain and the spatial locations of its generators were compared with those of the best investigated evoked responses of the auditory system the slow auditory evoked responses (sAER). We will report here results from three different sets of experiments: the study of stimulus repetition rate, of tonotopy (dependence upon stimulus frequency) and of attentional variations.

\section{METHODS}

\section{Subjects}

A total of 12 subjects with no history of otological or neurological disorders and normal audiological status, aged between 23 and 42 , participated in the experiments. All subjects were right-handed. Informed consent was obtained from the subjects after the nature of the particular experiment was fully explained to them.

\section{Stimulation}

Stimuli were delivered to the right ear. They were presented through a nonmagnetic and echo-free stimulus delivery system with an almost linear frequency characteristic (deviations less than $\pm 4 \mathrm{~dB}$ in the range between 200 and $4000 \mathrm{~Hz}$ ). During stimulus presentation the subjects were asked to stay awake and to keep their eyes open.

The recovery function of the GBR and of the SAER was studied in four subjects at stimulus rates of $1 / 8,1 / 4,1 / 2$ and $1 \mathrm{~Hz}$, interstimulus intervals of $8,4,2$ and $1 \mathrm{~s}$, respectively. Stimuli were Gaussian tone-pulses with half-value time of $6 \mathrm{~ms}$, a carrierfrequency of $1000 \mathrm{~Hz}$, and an intensity of $60 \mathrm{~dB} \mathrm{nHL}$ (normative hearing level). They were presented in two blocks of 256 stimuli each.

The dependence upon the stimulus frequency of the GBR and the sAER, characterizing one of the most important features of the auditory system - its tonotopic organization - was studied in two subjects to stimulus frequencies of 250,1000 and $4000 \mathrm{~Hz}$. Stimuli were tone burst of $500 \mathrm{~ms}$ duration, $15 \mathrm{~ms}$ rise and fall times, $4 \mathrm{~s}$ interstimulus interval, $60 \mathrm{~dB} \mathrm{nHL}$ intensity and the above mentioned carrier frequencies. They were presented in three blocks of 128 stimuli each. 
- Attend Condition

- odd-ball paradigm (33\%:67\%)

- counting low tones

- Distract Condition

- Letter \& Number Task

Figure 1. Stimulus design of the odd-ball paradigm: probability of appearance of the rare stimuli (low pitch) has $33 \%$, of the frequent stimuli (high pitch) $67 \%$. During the attend condition the subject concentrated on the appearance of the rare stimuli and had to count them. During the distract condition the subject performed a letter-to-number test (example shown above), involving mental arithmetic and considerable demand on memory.

Effects of attention and processing on the GBR and the sAER was studied in six subjects. In a classical odd-ball paradigm rare stimuli (low pitch of $667 \mathrm{~Hz}, 33 \%$ of all stimuli) were randomly interspersed in a sequence of frequent stimuli (high pitch of $1500 \mathrm{~Hz} ; 67 \%$ ). Stimuli were Gaussian tone-pulses of the above mentioned frequencies with half-value time of $6 \mathrm{~ms}$ and an intensity of $60 \mathrm{~dB}$ nHL. The interstimulus interval varied randomly from 1.5 to $2.5 \mathrm{~s}$. The stimuli were presented in twelve blocks, with one block having a random length between 150 and 250 stimuli. During one block, the subjects task was either to attend to the oddball stimuli or to process a completely different task in order to distract from the oddball stimuli. During the attend condition, the subject was instructed to concentrate on the appearance of rare stimuli and to count them. The counting results were controlled. During the distract condition, the subject had to perform a numbers-to-letter task involving mental arithmetic with considerable demand on memory (c.f. Fig. 1). With the begin of the stimulus block (distract condition), the subjects started to calculate the sum of the numbers corresponding to the rank of the letters of certain well known sentences. The results of their calculations were also controlled. Task-sequence was randomized across blocks.

\section{Neuromagnetic recordings}

Recordings were carried out in a magnetically shielded room using a 37 channel SQUID (Super Conducting Quantum Interference Device) biomagnetometer (Biomagnetic Technologies). Detection coils of the biomagnetometer are arranged in a circular concave array with a diameter of $144 \mathrm{~mm}$, and a spherical radius of 122 $\mathrm{mm}$. The axes of the detection coils are normal to the surface of the sensor array. The distance between the centers of two adjacent coils is $22 \mathrm{~mm}$; each coil itself measures $20 \mathrm{~mm}$ in diameter. The sensors are configured as first-order axial gradiometers with a baseline of $50 \mathrm{~mm}$. The spectral density of the intrinsic noise of each channel is between $5-7 \mathrm{fT} / \sqrt{\mathrm{Hz}}$ in the frequency range above $1 \mathrm{~Hz}$. The subjects lay in a right lateral position with their head, neck and upper part of the body supported by a specially fabricated vacuum cushion. A sensor position indicator system determined the spatial locations of the sensors relative to the head and indicated if head movements occurred during the recordings. The sensor array was centered over a point about $1.5 \mathrm{~cm}$ superior to the position T3 of the 10-20 system for electrode 
placement and was positioned as near as possible to the subject's head. Using a band width from 1 to $100 \mathrm{~Hz}$ and a sampling rate of $520.8 \mathrm{~Hz}$ (16 bit ADC), stimulusrelated epochs between 1000 and $1200 \mathrm{~ms}$ for the different experiments (including $200 \mathrm{~ms}$ prestimulus baselines) were recorded and stored for further analysis. The slope was $6 \mathrm{~dB} /$ oct for the analog high-pass filter and $60 \mathrm{~dB} /$ oct for the on-line digital low-pass filter.

\section{Data Analysis}

Wide band responses to each block were first averaged after rejection of artifact-contaminated epochs due to eye blinks or muscle activity. Since the waveforms of the averaged responses recorded in the different blocks were highly reproducible, their grand average was used for further evaluation. The wide-band responses were digitally filtered with passbands $0.1-20 \mathrm{~Hz}$, and $24-48 \mathrm{~Hz}$ (first order Bessel filter, $6 \mathrm{~dB} / \mathrm{oct}$ ) in order to obtain the slow auditory evoked field (sAEF) and the gammaband field (GBF), respectively. To avoid phase shifts, the data were filtered twice (forward and backward); thus, the effective slope of the filter characteristic was 12 $\mathrm{dB} /$ octave. Root-mean-square (RMS) field values over the 37 recording channels were calculated for every sampling point.

Source analyses based on a single moving Equivalent Current Dipole (ECD) model in a spherical volume conductor (Sarvas, 1987) was applied to both magnetic field distributions studied: bandwidths $0.1-20 \mathrm{~Hz}$ and $24-48 \mathrm{~Hz}$. The size of the sphere was determined by a fit to the scalp in the area of measurement. It was in this area that the shape of the scalpwas digitized with the sensor position indicator. The center and the radius of the best fitting sphere were determined using a least squares fit algorithm. The relative angulation of the pickup coils and the influence of the volume currents were taken into account for the source analysis. Source locations, their confidence volumes and the dipole moment were estimated for each sampling point in a head-based coordinate system. The origin of this coordinate system was set at the midpoint of the medial-lateral axis (y-axis) which joined the center points of the entrance to the acoustic meatuses of the left and the right ears (positive towards the left ear). The posterior-anterior axis ( $\mathrm{x}$-axis) was oriented from the origin to the nasion (positive towards the nasion) and the inferior-superior axis (z-axis) was perpendicular to the $x$-y plane (positive towards the vertex).

The estimated source locations ( $x, y, z$ coordinates) of the major wave of the sAEF - N1m and of the three largest peaks of the transient GBF were determined by means of the following manipulations. For each wave-peak the time interval (hereafter termed "evaluation interval") containing the field maximum and minimum, the RMS field maximum, and the goodness of fit between the theoretical field generated by the model and the measured field were determined. The spatial ECD coordinates $(x, y, z)$ for the $\mathrm{N} 1 \mathrm{~m}$ wave of the SAEF were assigned to the corresponding averaged values of seven adjacent sampling points around the center of the obtained evaluation interval. For the GBF, the "evaluation interval" was determined for each of the three largest peaks (number of adjacent sampling point usually 3-4) and the corresponding averaged values were averaged across the three peaks. However, only those $\mathbf{x}, \mathbf{y}$ and $z$ values were included in the average which further fulfilled the following conditions based on statistical and anatomical considerations: 
- goodness of fit above $95 \%$ for the N1m wave of the SAEF and above $90 \%$ for the GBF

- distance of the ECD to the midsagittal plane more than $2 \mathrm{~cm}$

- inferior-superior value greater than $1 \mathrm{~cm}$

- confidence volume of the source location smaller than $0.3 \mathrm{~cm}^{3}$
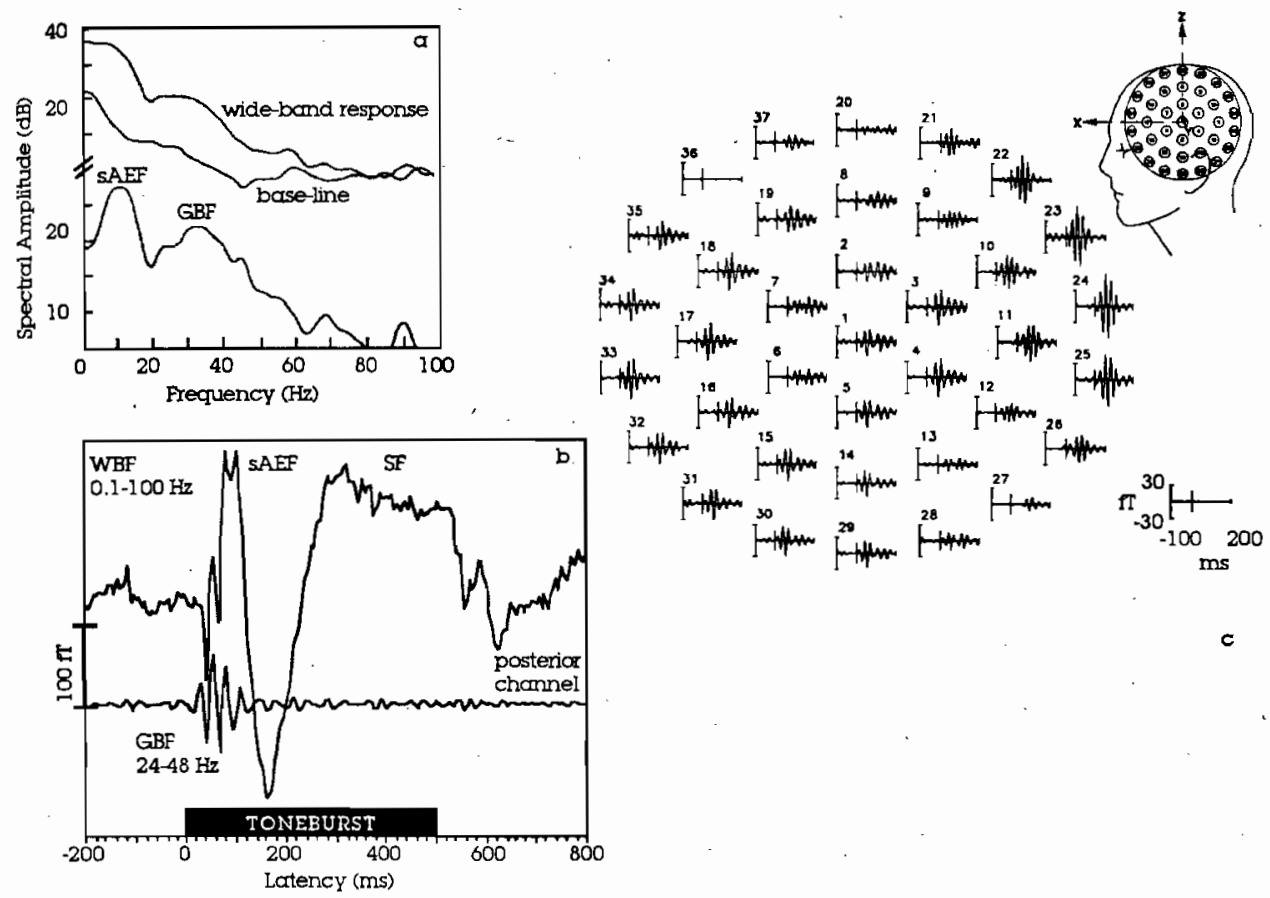

Figure 2. (a) Spectrum of the wide-band responses averaged over three consecutive data blocks from each of seven subjects. The top two traces were constructed by averaging the Fourier amplitude spectra of $200 \mathrm{~ms}$ Hanning-windowed epochs and smoothing the result with a rectangular $3 \mathrm{~Hz}$ window. Epochs selected were ( -35 to $165 \mathrm{~ms}$ ) for the wide-band response (top trace), and (-200 to $0 \mathrm{~ms}$ and 300 to $500 \mathrm{~ms}$ ) for the baseline estimate (middle trace). The bottom trace is the log difference between the response and baseline spectra, clearly showing separate spectral peaks for the slow auditory evoked field (sAEF) and the gamma-band field (GBF) components. (b) Auditory evoked gammaband field as compared to the corresponding wide-band field (WBF). The coincidence of GBF peaks in the unfiltered and filtered data is visible. (c) Auditory evoked gamma-band field distribution over the left hemisphere $(\mathrm{N}=384)$.

\section{RESULTS}

Fig. 2a shows a spectrum grand-averaged across 384 stimulus-related epochs for each of the 37 magnetic channels over the supratemporal cortex for 7 different subjects in response to $500 \mathrm{~ms}$ tone-burst stimulation. The wide-band response contains spectral energy in two distinct frequency ranges. One peak around $10 \mathrm{~Hz}$ representing the sAEF, and another one between $30-40 \mathrm{~Hz}$ reflecting the GBF. A smaller peak at around $70 \mathrm{~Hz}$ also seems evident but will not be examined here any further. 
To extract the time function of the transient oscillatory gamma-band response from the wide-band activity, responses were digitally filtered with a $24-48 \mathrm{~Hz}$ pass-band. An example of the wide-band $(01 .-100 \mathrm{~Hz})$ and the gamma-band response (24$48 \mathrm{~Hz}$ ) is shown in Fig. 2b. In the wide-band response not only the large slow wave components but also noticeable gamma-band activity during the first $100 \mathrm{~ms}$ after the stimulus onset can be clearly discerned. The sustained field and the "off" response can also be well detected. Finally, this figure represents the excellent coincidence between the GBF peaks in the wide-band and in the narrow-band version of the data. Fig. $2 \mathrm{c}$ displays the gamma-band field distribution after the tone-burst stimulation, as obtained through the 37 recording magnetic channels. The field distribution shows field minimum and maximum at similar locations as the SAEF, suggesting a source of the GBF in the supra-temporal cortex as well. The gamma-band field has clear dipolar pattern, thus a single moving ECD model can be used in order to estimate the underlying sources. It was shown by (Pantev et al., 1991), that the GBF equivalent source is located in cortical regions on the floor of the Sylvian fissure (Brodman's areas 41, 42 and 22), more medial and anterior than the source location of the major N1m-component of the SAEF.

a slow AEF (0.1-20 Hz)

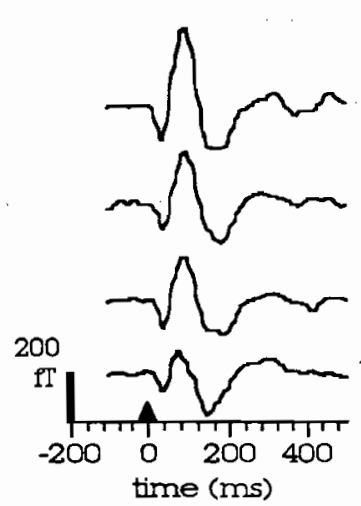

b $\mathrm{GBF}(24-48 \mathrm{~Hz})$

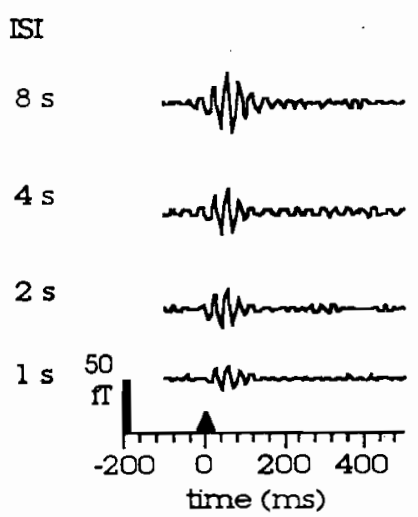

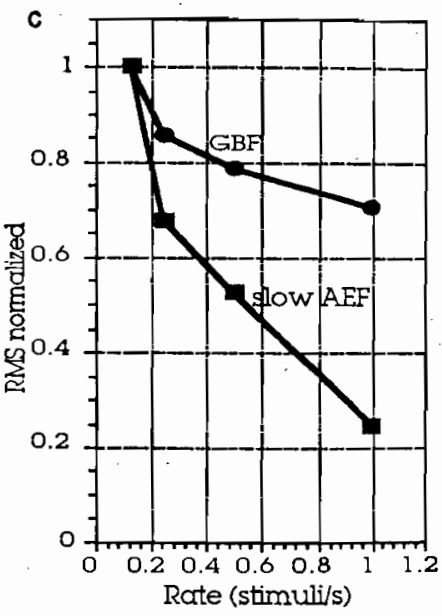

Figure 3. Experimental set of data for four different interstimulus intervals of 8, 4, 2, and $1 \mathrm{~s}$. (a) Slow. auditory evoked fields (b) Gamma-band fields. (c) This panel indicates the rate-amplitude-characteristics of the slow AEF and GBF based on the mean RMS-values averaged across subjects as relative presentation referred to the maximum value obtained at ISI of $8 \mathrm{~s}$.

Figures $3 a, b$ illustrate the $\mathrm{SAEF}$ and the GBF at the different 4 rates with interstimulus intervals of $8,4,2$ and $1 \mathrm{~s}$. The appearance of the stimulus is marked by a filled triangle on the abscissa. The amplitude of $\mathrm{N} 1 \mathrm{~m}$ of the SAEF and this one of the GBF appear to decrease as the stimulus rate increases; however, the N1m is decreasing faster than the GBF. In order to estimate the recovery function of the GBF and the $\mathrm{N} 1 \mathrm{~m}$, the dependence of the RMS field amplitude calculated over the $37 \mathrm{mag}$ netic channels and averaged across subjects, upon the stimulus rate (termed "rate- 
amplitude characteristic") was determined. Because of the very different magnitudes of N1m and the GBF, the comparison of their characteristics is proper after they have been normalized. The normalized "rate-amplitude characteristics", which are shown in Fig. 3c, have different slopes, indicating different recovery patterns as well different generation mechanisms of the sAEF and the GBF.

Using the source localization procedure described in the Methods section, the equivalent source locations for the $\mathrm{N} 1 \mathrm{~m}$ and the GBF were determined for each stimulus rate. The consistency of the localization results across subjects allowed calculation of the grand means of the estimated source locations for each stimulus rate, as shown in Fig. 4.

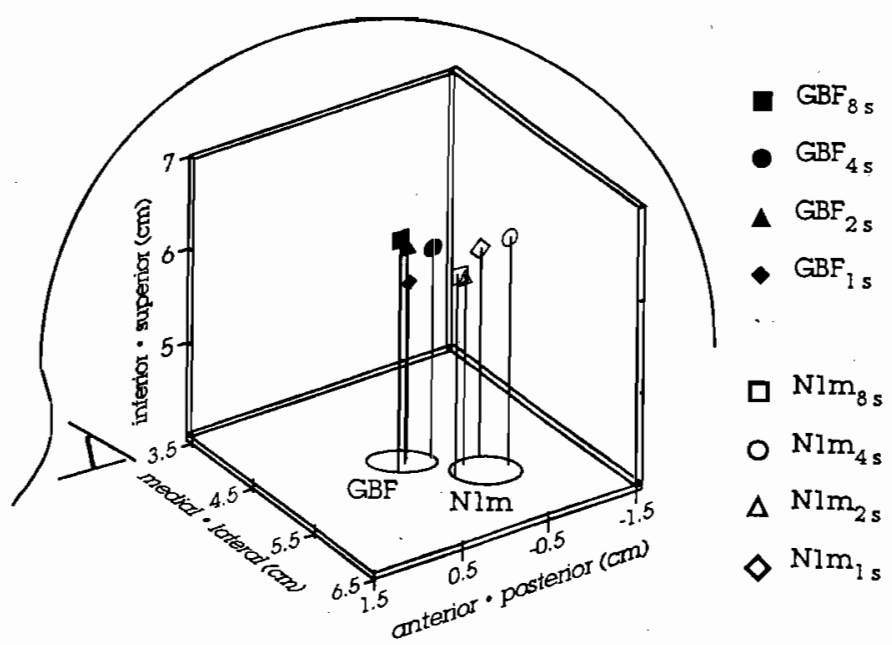

Figure 4. Mean values of the estimated source locations of the slow AEF and GBF for the different interstimulus intervals. Anterior-posterior, medial-lateral and inferior-superior axes of the 3D-plot represent the axes of the head-based coordinate system as described in the Methods section.

Two separate groups of source locations can be clearly recognized. The first one (open symbols) includes the locations of $\mathrm{N} 1 \mathrm{~m}$; this group is most lateral and posterior. The second group (filled symbols) represents the mean locations of the GBF, also suggesting a source in the supra temporal cortex somewhat medial and anterior to $\mathrm{N} 1 \mathrm{~m}$. The data suggest that the GBF and the SAEF have different origins and may arise from different processes in the auditory pathway.

The tonotopy is one of the general principles of functional organization of the auditory system (Romani et al., 1982; Pantev et al., 1988; Pantev et al., 1989; Bertrand et al., 1991). The depth of the N1m source indicates this tonotopic organization: the higher the stimulus frequency, the deeper the equivalent source. Moreover, in most subjects the depth of $\mathrm{N} 1 \mathrm{~m}$ increases linearly with the logarithm of the stimulus frequency (Fig. 5, upper part). Opposite to this finding for the $\mathrm{N} 1 \mathrm{~m}$ component of the SAEF was that the depths of the equivalent GBF source (Fig. 5, 
lower part) obtained from the same data sets of the two investigated subjects do not change with an increase in the stimulus frequency.

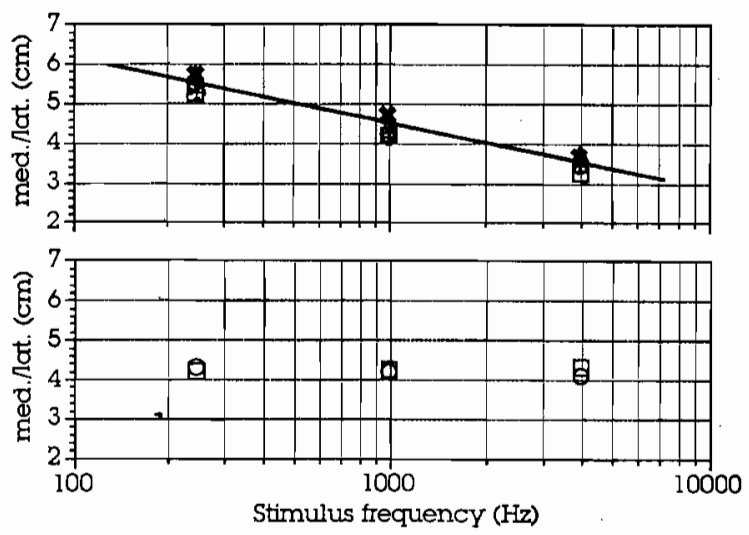

Figure 5. Dependence of the y-coordinate of the source location (corresponding to the depth of the equivalent source) of the N1m of the slow AEF (top) and the GBF (bottom) upon the stimulus frequency $(250,1000$ and $4000 \mathrm{~Hz})$ as obtained from two different subjects.
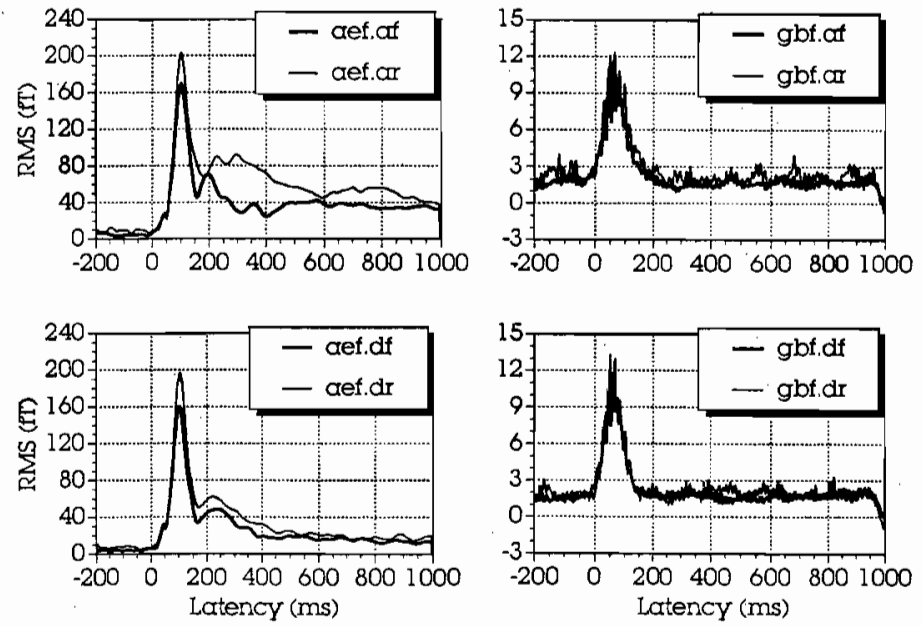

Figure 6. RMS field values over the 37 recording channels and grand averaged across subjects for the rare (thin line) and the frequent (thick line) stimuli of the attend (top) and distract (bottom) conditions for the slow AEF (left) and the gamma-band field (right). af - attend condition, frequent stimulation; ar - attend condition, rare stimulation; $\mathrm{df}$ - distract condition, frequent stimulation; $\mathrm{dr}$ - distract condition, rare stimulation

The results of the attention experiment for the "odd-ball" stimulus paradigm are illustrated in the next two figures. Fig. 6 shows the RMS field values over the 37 
recording channels, which were averaged across the six subjects investigated and which are displayed separately for the rare stimuli (thin line) and frequent stimuli (thick line) of the attend condition (top panels) and distract condition (bottom panels) for the SAEF (left side) and the GBF (right side). A clear difference was obtained within the time-range of P300 for the attend and distract conditions, indicating that the subjects complied well with the experimental task. Higher amplitudes of $\mathrm{N} 1 \mathrm{~m}$ were obtained for the rare stimuli in both attend and distract conditions. No substantial difference was obtained for the GBF, neither between the rare and frequent stimuli, nor between the attend and distract conditions.
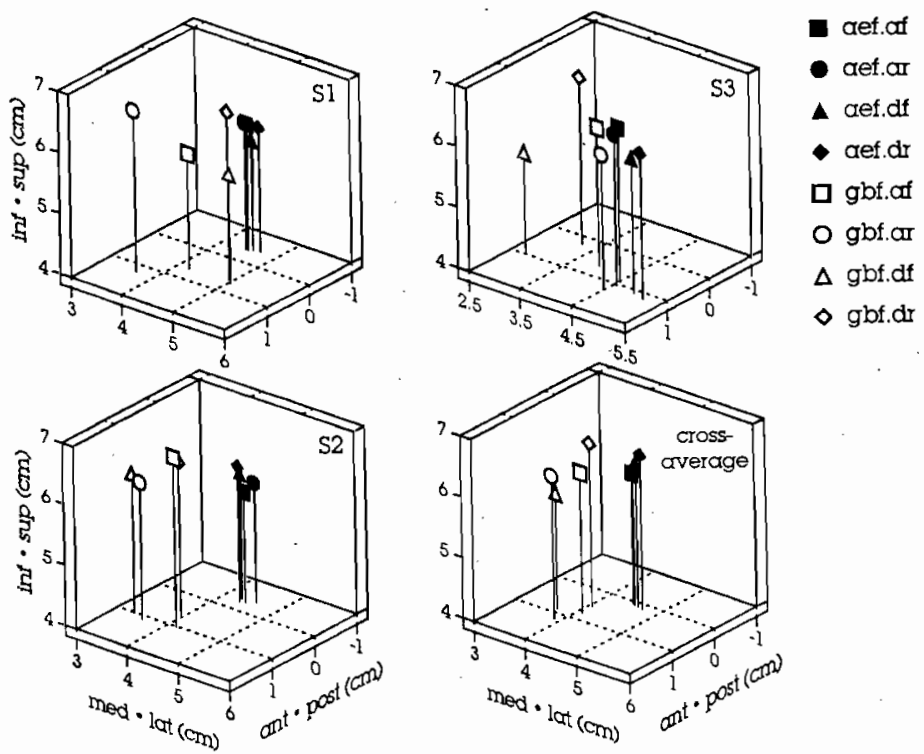

Figure 7. 3D-source locations of the N1m of the slow AEF and the GBF for the different conditions: af, ar, df, and dr (c.f. Fig. 6) as obtained from three different subjects (S1, S2, S3). The right low panel shows the cross-average source locations obtained from all subjects investigated. Anterior-posterior, medial-lateral and inferior-superior axes represent the axes of the head-based coordinate system as described in the Methods section.

The results of the corresponding source analysis of N1m and the GBF for rare and frequent stimuli as well as attend and distract conditions are presented in Fig. 7 for the 3 best subjects (S1, S2, and S3 were the subjects with the best signal-to-noise ratio). The grand mean of the corresponding source locations of the six subjects are shown at the bottom right corner. As already demonstrated by Fig. 4 the GBF sources are more anterior and more medial than those of $\mathrm{N} 1 \mathrm{~m}$. Neither the N1m nor GBF differed in source location between conditions (rare/frequent or attend/distract comparisons by means of paired t-test) 


\section{DISCUSSION}

The auditory evoked gamma-band response seems to be an event related rhythmic response which persists within the first 100 to $120 \mathrm{~ms}$ after the stimulus onset. The individual waves of the GBF are visible in the subject's wide-band unfiltered data and show a clear spectral peak between 30 and $40 \mathrm{~Hz}$. After separation of the wide-band activity in $\mathrm{SAEF}$ and GBF, the moving single equivalent current dipole model accounts for each data set (sAEF and GBF) almost completely. The obtained source locations for the $\mathrm{AAEF}$ and the GBF differ significantly, but both responses originate within the supratemporal cortex. The GBF sources for the different stimulus rates were found to be medial and anterior to the sources of the $\mathrm{N} 1 \mathrm{~m}$ of the sAEF.

If the N1m and GBF, two components of the complex human auditory cortical response, represent responses to information of the same aspects of the auditory pathway, then their rate-amplitude characteristics should also be similar. The pronounced difference in the slopes of their rate-amplitude characteristics, however, indicates different recovery patterns and, hence, different generator mechanisms for the N1m and GBF. The finding of separate habituation patterns for the auditory $\mathrm{N} 1 \mathrm{~m}$ and GBF is paralleled by the results of an investigation of slow and gammaband evoked responses in the somatosensory system (Kaukoranta and Reinikeinen, 1985).

Tiitinen et al. (1993) investigated the effect of selective attention on the transient auditory gamma-band potentials by having subjects listen to tone pips presented in one ear while ignoring concurrent sequence of tone pips in the other ear. They found that the gamma-band response was significantly larger when subjects paid attention to stimuli rather than when they ignored them and that this attention effect was most pronounced over the frontal and the central scalp areas. This attention effect was found only for the frequency band with central frequency near $40 \mathrm{~Hz}$. The authors thus concluded that this transient auditory gamma-band response demonstrates a physiological correlate of selective attention in humans. In our manipulation of global attention, we were not able to show a variation of the transient auditory GBF upon attentional variables neither in the GBF-amplitude in time domain, nor in the GBF-source location. A possible explanation for this difference seems to be the technical details: Tiitinen et al. (1993) used EEG-recordings and a much smaller frequency band ( $8 \mathrm{~Hz}$ around the central frequency of $40 \mathrm{~Hz}$ ) as compared to the $24 \mathrm{~Hz}$ band (24-48 Hz) of MEG used in our studies. More likely, however, the difference in experimental conditions may have caused the different outcome: Tiitinen et al. (1993) varied selective attention, which means that a specific subset of stimuli must be processed while another subset should be ignored, whereas the present experiment included a variation in global attention, meaning that all of the incoming stimuli must be attended in one case or were ignored in the other condition.

The experimental results of the auditory evoked, time and phase locked gamma band activity presented in this chapter allow us to make the following speculations: The different recovery pattern of the SAEF and the GBF as well as their different origins point out that these two different kinds of cortical activity may have different generator mechanisms and may arise from different steps in the course of processing of auditory information. This view is supported by the present results of different 
short-term habituation for the sAEF and the GBF and receives additional confirmation by the difference in the dependence on the stimulus repetition rate of both phenomena. Whereas the slow auditory evoked activity is probably representing cortical analysis of auditory stimulus features, the missing tonotopic representation in the GBF source locations lets us assume that the gamma-band activity may represent more general perception mechanisms in the central part of the auditory system. Due to the fact that gamma-band activity appears relatively early and does not demonstrate cortical tonotopic representation, it could be concluded that the gamma-band activity reflects a cortical process synchronizing different auditory areas. According to the suggestions outlined by Roelfsema et al. and Eckhorn (see respective chapters in this volume) this more global cortical synchronization could serve as a dynamic link of separate subregions of the auditory cortex in order to combine complex auditory features into unitary auditory percepts, which can also be regarded as a kind of recognition of auditory objects. Synchronizing activity is also predicted by models of brain functioning (e.g. Pulvermüller et al., this volume). Grossberg $(1980,1987)$ for example suggests that a dynamic state which he refers to as "adaptive resonance" occurs between successive stages of processing. Such a short-term memory resonance is needed to drive changes in long-term memory and might express itself as gamma-band activity. Therefore, the relationship of the GBF to memory functions should be explored in future experiments.

\section{Acknowledgement}

Research was supported by the Deutsche Forschungsgemeinschaft. We appreciate the assistance of and critical discussions with many colleagues and friends, in particular of Olivier Bertrand, Carsten Eulitz, Scott Hampson, Bernd Lütkenhöner, Scott Makeig, Brigitte Rockstroh, and Bernhard Roß.

\section{REFERENCES}

Basar, E. A study of the time and frequency characteristics of the potentials evoked in the acoustical cortex. Kybernetik, 1972, 10: 61-64.

Basar, E. EEG-Dynamics and evoked potentials in sensory and cognitive processing by the brain. Berlin-Heidelberg-New York: Springer-Verlag, 1988, 1:311-318.

Basar, E. and Özesmi, C. The hyppocampal EEG activity and a systems analytical interpretation of averaged evoked potentials of the brain. Kybernetik, 1972, 12: 45-54.

Basar, E., Rosen, B., Basar-Eroglu, C. and Greitschus, F. The associations between $40 \mathrm{~Hz}$-EEG and the middle latency response of the auditory evoked potential. Int. J. Neurosci, 1987, 33: 103-117.

Basar, E. and Ungan, P. A component analysis and principles derived for the understanding of evoked potentials of the brain: Studies of the hyppocampus. Kybernetik, 1973, 12: 133-140.

Basar-Eroglu, C. and Basar, E. A compound $\mathrm{P} 300-40 \mathrm{~Hz}$ response of the cat hippocampus. Int. J. Neuroscience, 1991, 60: 227-237.

Bertrand, O., Perrin, P. and Pernier, J. Evidence for a tonotopic organization of the auditory cortex observed with auditory evoked potentials. Acta Otolaryngol (Stockh), 1991, Suppl. 491:116-123.

Crossberg, S. How does a brain build a cognitive code? Psychol. Rev., 1980, 87: 1-51.

Crossberg, S. The adaptive brain. Amsterdam: Elsevier, 1987, I. and II.

Eckhorn, R. Oscillatory and non-oscillatory synchronizations in the visual cortex of the cat and monkey. (this volume). 
Elbert, T. Slow cortical potentials reflect the regulation of cortical excitability. New York, London: Plenum Publishing Corp., 1993, 235-252.

Galambos, R. and Makeig, S. Dynamic changes in steady-state responses. Berlin: Springer, 1988, 103122.

Galambos, R., Makeig, S. and Talmachoff, P.J. A 40-Hz auditory potential recorded from the human scalp. Proc. Natl. Acad. Sci, 1981, 78: 2643-2647.

Hari, R., Hämälainen, M. and Joutsiniemi, S.L. Neuromagnetic steady-state responses to auditory stimuli. J Acoust Soc Am, 1989, 86: 1033-9.

Kaukoranta, E. and Reinikeinen, K. Somatosensory evoked magnetic fields from SI: An interpretation of the spatiotemporal field pattern and effects of stimulus repetition rate. Helsinki University of Technology, Low Temperature Laboratory, 1985

Makeig, S. and Galambos, R. The $40-\mathrm{Hz}$ band evoked responses lasts $150 \mathrm{msec}$ and increases in size at slow rates. Phoenix 9.30.89: 1989, 15: 113.

Mäkelä, J.P. and Hari, R. Evidence for cortical origin of the $40 \mathrm{~Hz}$ auditory evoked response in man. Electroencephalogr Clin Neurophysiol, 1987, 66: 539-46.

Pantev, C., Elbert, T., Makeig, S., Hampson, S., Eulitz, C. and Hoke, M. Relationship of transient and steady-state auditory evoked fields. Electroenceph. clin. Neurophysiol., 1993, 88: 389-396.

Pantev, C., Hoke, M., Lehnertz, K., Lütkenhöner, B., Anogianakis, G. and Wittkowski, W. Tonotopic organization of the human auditory cortex revealed by transient auditory evoked magnetic fields. Electroenceph. clin. Neurophysiol., 1988, 69: 160-170.

Pantev, C., Hoke, M., Litkenhöner, B. and Lehnertz, K. Tonotopic organization of the auditory cortex: pitch versus frequency representation. Science, 1989, 246: 486-8.

Pantev, C., Makeig, S., Hoke, M., Galambos, R., Hampson, S. and Gallen, C. Human auditory evoked gamma-band magnetic fields. Proc Natl Acad Sci, 1991, 88: 8996-9000.

Ribari, U., Llinas, R., Kluger, A., Suk, J. and Ferris, S.H. Neuropathological dynamics of magnetic, auditory steady-state responses in Alzheimer's disease. New York: Plenum Press, 1989, 311-314.

Roelfsema, P.R., Engel. A.K., König, P., Singer, W. Oscillations and synchrony in the visual cortex: evidence for their functional relevance. (this volume).

Romani, G.L., Williamson, S.J., Kaufman, L. and Brenner, D. Characterization of the human auditory cortex by the neuromagnetic method. Exp Brain Res, 1982, 47: 381-93.

Sarvas, J. Basic mathematical and electromagnetic concepts of the biomagnetic inverse problem. Phys. Med Biol., 1987, 32: 11-22.

Tiitinen, H., Sinkkonen, J., Reinikeinen, K., Alho, K., Lavikeinen, J. and Näätảnen, R. Selective attention enhances the auditory 40-Hz transient response in humans. Nature, 1993, 364: 59-60. 